INTRODUCTION

\title{
The FIFA/F-MARC update on doping
}

\section{J Dvorak, P McCrory, M D'Hooge, M Saugy}

Br J Sports Med 2006;40(Suppl I):i2. doi: 10.1136/bjsm.2006.027797

T he ongoing media debate surrounding the issue of doping in sport has raised public awareness of a problem that been steadily developing over many years. This controversy reflects both the rapid development of various sports disciplines as well as the evolution of new doping methods and agents.

The Fédération Internationale de Football Association (FIFA) introduced doping controls in football in 1970 to ensure that the results of national and international matches were a fair reflection of the ability of those taking part. Over the past 12 years, the FIFA Medical Assessment and Research Centre (F-MARC) has developed a worldwide network of specialists who are involved in the educational process within the regional football confederations and national associations as one facet of global anti-doping strategies. F-MARC has also been involved in the practical implementation of doping controls for FIFA competitions at all levels. FIFA has developed close collaboration not only with the confederations and member associations, but also with other team sports federations, particularly with the accredited drug testing laboratories. These relationships have helped to understand the extent of doping, which in turn forms the basis of a global, harmonised strategy in the fight against doping in football.

Football has approximately 200000 elite players worldwide and more than 20000 doping controls are performed on these players each year. The incidence of positive tests accounts for $0.4 \%$ of all tests performed. The commonest doping cases are for so-called recreational or social drugs (such as cannabis and cocaine) and only $0.07 \%$ of all cases are linked to the misuse of anabolic steroids. Analysis of these findings, including the distribution of positive cases among the FIFA confederations, determines the nature and direction of the ongoing FIFA/F-MARC strategy in the fight against doping.

In this supplement, the first paper describes the background, historical perspectives and the development of the FMARC strategy in the fight against doping. The following articles are structured in a way that they reflect information according to the frequency of positive cases-that is, cannabis, cocaine, testosterone, and synthetic anabolic steroids. Nandrolone deserves special attention as many food supplements sold over the counter are contaminated by undeclared nandrolone or nandrolone precursors which thus may be consumed by players without their knowledge. However, it is clear that all players are responsible for their diet, including the intake of food supplements and vitamins.

The papers on erythropoietin, blood doping and human growth hormone offer detailed information about substances that are frequently discussed in the media. Over the past three years, however, not a single football player has tested positive for erythropoietin and/or blood doping.

Football players who have acute or chronic diseases or physical symptoms and signs following an injury may need to be treated with specific pharmacological preparations which may be banned under the prohibited list of substances. In such cases, a therapeutic use exemption (TUE) may be granted if clinically justified. The commonest applications for TUE in FIFA are for $\beta 2$ agonists (for treatment of asthma) and corticosteroids (for treatment of acute injuries). Both groups of drug are discussed in detail in separate papers.

In the management of positive samples, FIFA follows the concept of "separation of power". Medical doctors analyse the medical circumstances of the player who has tested positive and the laboratory estimates the severity of violation of the FIFA doping regulations in relation to the prohibited substance found. It is then the duty of the FIFA Doping Control Sub-Committee to present the medical and laboratory aspects of a positive case to the Disciplinary Committee of FIFA. The last article deals with the medicolegal aspects of the management of positive cases.

Despite the fact that the incidence of positive doping cases among football players is low in comparison with other sports (according to the 2004 statistics of the World Anti-Doping Agency), FIFA is convinced that a stringent system of doping controls is critical to the future of the sport. However, FIFA also acknowledges that the education of players, and coaches and paramedical and medical personnel in contact with football players is likely to be even more important in the fight against drugs in sport and creating a culture that recognises that doping has no place in football.

This 2006 update on the FIFA strategy in the fight against doping in football should contribute to the understanding of the problem and is an integral part of the long term educational programme of FIFA.

\section{Authors' affiliations}

J Dvorak, FIFA Chief Medical Officer, FIFA Medical Assessment and Research Centre, and Department of Neurology, Schulthess Clinic,

Zurich, Switzerland

P McCrory, Centre for Health, Exercise and Sports Medicine, University of Melbourne, Australia

M D'Hooghe, FIFA Sports Medical Committee, FIFA Executive

Committee, Bruges, Belgiun

M Saugy, Swiss Laboratory for Doping Analyses, Institute of Legal

Medicine, Lausanne, Switzerland

Correspondence to: Professor J Dvorak, Chairman, FIFA Medical Assessment and Research Centre, Department of Neurology, Schulthess Clinic, Lengghalde 2, 8008 Zurich, Switzerland; jiri.dvorak@kws.ch 